\title{
Melkart y el politeísmo tirio. Apostillas al trabajo de C. Bonnet (1988)
}

\author{
Franciso Javier Burgaleta Mezo
}

Parece sobrado reescribir sobre Melkart tras haber aparecido un trabajo tan completo y exhaustivo como el de C. Bonnet (1988), como bien se ha reconocido ${ }^{1}$.

La obra de la investigadora belga es efectivamente modélica. Destaca en particular la completa recopilación de información que se presenta en un corpus documental bien trabado, abundantemente anotado y con aparato bibliográfico extenso. Se ofrece con ella, tanto a especialistas como a estudiosos de otras ramas interesados por diferentes motivos en la divinidad, una herramienta que supone un avance definitivo en el tratamiento de la figura. Por ello merece todos los elogios y pocos comentarios más ${ }^{2}$.

\footnotetext{
1 Marcel LE GLAY: 1989, Mythes, légendes et réalites cultuelles. Latomus XLVIII, fasc 3, pp. 581-589.

2 En cualquier caso, las pequeñas observaciones que pudieran hacerse sobre puntos concretos, como la problemática atención a la presencia en la Magna Grecia (BONNET 1988: p. 307), o Panonia (id. p. 312), se explican en la intención de abarcar toda la documentación efectivamente "posible" 0 siquiera "probable". En el caso de Panonia consideramos que, pese a la afirmación de J. FITZ (Sanctuaires d"Hercule en Pannonie, en Hommages Grenier, Bruselas, pp. 623-38), seguida por la autora, ni la documentación, ni la presencia que esta documenta, es mayor que en otras regiones, como la Galia, o en Germania. Para demostrar tal punto podemos remitirnos a los diferentes trabajos que tratan la figura, desde la de J. TOUTAIN (1917-18 (reimp. 1967), Les cultes paiens dans l'Empire romain, Paris, (ed. anastática), ed. L'Erma Roma) a E. THEVENot (THEVENot, Emile, 1968, Divinites et sanctuaires de la Gaule, Paris). Otros puntos más conflictivos o discutidos, y que pueden dar pie a otras interpretaciones, como son la presencia en la península itálica, en la que hemos emitido parcialmente nuestra opinión (BURGALETA MEZO, F.J., 1990, Los origenes y la naturaleza del Hércules romano, I Encuentro Coloquio de ARYS. (Jarandilla de la Vera, Cáceres, diciembre de 1989) sosteniendo una hipótesis todavía por terminar de aquilatar, como es el posible préstamo del modelo iconográfico en su llegada a la Península itálica (BuRgaleta Mezo, F.J., 1988, Un tipo iconográfico de Hércules en Occidente, Espacio, Tiempo y Forma (serie II, Historia Antigua, t. 1), Homenaje al profesor Eduardo Ripoll Perelló, pp. 223-32; y BuRgaleta Mezo y LUCAS Pellicen, 1993. Del tipo del "dios que golpea" y las estatuas "promachos" en la Peninsula itálica, en Bronces antiguos y religion romana, Actas del XI Congreso Intemacional de Bronces antiguos, Madrid, mayo-junio 1990: Madrid, CSIC, pp. 101-31, donde se presenta la bibliografia tratada).
} 
Pero este dossier es, como afirma la misma autora ${ }^{3}$, el punto de partida para iniciar cualquier reflexión. $Y$ como así mismo señala ${ }^{4}$, tras esa primera parte recopilativa, hay una segunda interpretativa y más comprometida ${ }^{5}$.

Para afrontarla anuncia al principio unos atinados presupuestos metodológicos. Se refiere así a una "interpretación global de los procesos históricos que han presidido la elaboración, la transformación y la alteración del personaje divino llamado Melkart» 6 ; y a una metodología de la historia de la religión, que quiere aclarar las singularidades históricas de las que cada civilización da pruebas en los recursos, la organización, etc. (BONNET, id: p. 8). Desde ahí acomete una construcción cupular, en la que todos los argumentos apuntan ${ }^{7}$ a una clave de tensión: explicar Melkart como divinización e hipóstasis del soberano, transformación basada en las figuras preexistentes de los rapaum o Refaim, ancestros reales divinizados, a los que alguna vez se alude como mlk o malikum: reyes. Tal reforma habría sido llevada a cabo por Hiram, primero en celebrar la egersis y campeón del expansionismo tirio. Aunque se reconoce una supuesta relación de Melkart con esa expansión tiria, tampoco se puede precisar en qué consistiría tal relación.

Es esencialmente sobre la consumación o no llevada a cabo de planteamientos de partida donde se introduce nuestro trabajo. Como sucede a menudo en historia, los complejos problemas metodológicos enunciados ( $y$ ya es importante que se reconozcan como tales) son difíciles de aplicar, y no siempre consiguen resolverse adecuadamente. La relación entre noticias religiosas puntuales no se contrasta con un análisis de las instituciones sociales, como la monarquía, cuando el argumento de la realeza sagrada es prioritario, y exige su disección para el ambiente concreto en que se sitúa el problema. Ni tampoco con las escasas, como siempre, noticias históricas que tenemos.

La escasez de material "directo" fenicio ${ }^{8}$ frente a la humana exigencia de interpretar, generan a veces posturas que, vistas desde fuera, no dejan

3 Recuérdese la afirmación de BONNET (1988: p. 10): "Sólo un sólido conocimiento del dossier autoriza a una formulación de hipótesis históricas, y no de juicios a priori".

4 BONNET 1988: p. 9: le plan adopté.

"Especulativa", la llama también (id. p. 9).

Cf. BONNET (1988) p. 9 y tambien en p. 417.

Diría "soportan" si el repaso que hace a la documentación efectivamente se fuese trabando en explicaciones construidas una sobre otra y enfocadas a resolver estas cuestiones; pero dada la enorme dificultad de enhebrar tan dispar conjunto de referencias más allá de la afirmación de su carácter poliado y nacional, cabria decir "eluden".

8 Este es un punto común en todas las consideraciones respecto de la religión fenicia: CAQUOT (1983) SZNYCER (1981 (a) y (b)), la misma BONNET (1988), etc. Se añaden además las dificultades de 
de resultar chocantes, como son los postulados. Por eso la atención a consideraciones globales resulta tan importante. Se trata de repasar los datos desde esa lógica cultural, entendiendo que es una construcción endeble ${ }^{9}$, sujeta a cualquier otra reconsideración procedente de nuevos datos, dada la dificultad manifiesta de generalizar sobre la religión fenicia (Caquot 1983; Xella 1986; Gras, Roulllard, Teixidor 1989: p. 35-6; y el largo etc). En suma, de entender la religión en una lógica conjunta con la historia y las instituciones.

La lectura repetida de la obra, trabada en algunas de las aportaciones elaboradas en los últimos decenios, nos ha producido algunas "sensaciones", si se nos permite el término ambiguo, que queremos anotar aquí. Son correcciones a lo que nos parecen en parte "percepciones de escuela» ${ }^{10}$ por un lado, y ciertas "contradicciones", vacios o elusiones en la presentación final de la figura. Comenzaremos para ello desde el mismo punto que C. Bonnet (id. p. 9) al referirse a los politeismos en general y a los fenicios en particular. Ahí insertaremos nuestra exposición, siguiendo los componentes de éstos - panteón, ritos, mitos- considerándolos como un conjunto, para contrastarlos luego con algunos aspectos de las realidades institucionales e históricas.

\section{POLITEISMO Y PANTEONES. EL CASO CANANEO, FENICIO Y TIRIO}

La relación de la religión con las restantes «estructuras de la realidad», siendo posibilista, es decir, mantiendo la primera una holgura al socaire de circunstancias históricas, la inserta en unos cauces que han de tenerse muy en cuenta. La Historia de las religiones, decíamos, ha de estar atenta tanto a las estructuras históricas como a los eventos concretos que en cada momento componen los primeros.

\footnotetext{
la lengua en su escasa conservación: 321 hapax legomena de 668 palabras identificadas en su vocabulario (RÓLLIG 1983: p. 376). El punto de contraste es la abundante documentación existente para la religión en Grecia y Roma, donde los problemas de organizarla primero, y de entenderla después, tampoco son livianos.

9 Tanto al menos como cualquier otra

10 En particular, en aquellos puntos de carácter general donde la figura se inserta en su contexto religioso y cultural. Se plantea con estas consideraciones, más o menos explicitadas en el texto de C. BONNET (ya que como veremos, se remite a las obras de otros autores, como P. XELLA), un trastondo de realidades que pueden desdibujar la figura. Por ejemplo, como anotaremos ahora, el tomar con tanto escrúpulo la posibilidad de existencia de tradiciones míticas en el mundo fenicio, termina por no considerar este aspecto en el tratamiento de Melkart, cuando hay varios testimonios de bastante peso como para sugerir tal posibilidad.
} 
Los "politeismos", entendidos como un estadio en las configuraciones religiosas asociado a unas realidades históricas determinadas (ZWI WERBLOWSKY 1987/b), como son las de la Antigüedad, forman parte de la existencia de áreas culturales relativamente comunicadas, y relativamente aisladas. Representan la plasmación de esa realidad con las paradojas y contradicciones consiguientes, parcialmente asumidas en la voluble manifestación de hierofanía que representan los dioses antropomorfizados ( $Z w 1$ WERBLOWSKY 1987/a) y personalizados; explicados en mitos etiológicos que se integran más o menos en una mitología general.

Este es un problema posiblemente central de los politeismos en las religiones antiguas, donde áreas culturales y unidades políticas entran en contradicciones relativas, al ser por una lado inevitable cierto grado de reconocimiento mutuo. Pero imposible una comunicación tan fluida entre cada componente del área cultural como para homogeneizarla, e inexistente un criterio de definición excluyente ${ }^{11}$.

La «encomiable» percepción de A. Brelich (1958) y M. Detienne (1986) usada por $\mathrm{C}$. Bonnet ${ }^{12}$ ha de ampliarse para hacer constatar estas excepciones y contradicciones en la lógica de tal sistema como consustanciales a ella ${ }^{13}$.

Por otra parte, la variedad de lo peculiar se integra dentro de unos cánones de pensamiento. La estructura social, política, institucional de este ambiente cananeo y fenicio es indispensable para entender semejante "lógica cultural» ${ }^{14}$. Desde ella los acontecimientos históricos concretos, de los que tan sólo conocemos un puñado, y que han podido improntar una realidad religiosa, han de valorarse en una imprescindible reflexión conjunta.

\subsection{Los politeismos cananeo y fenicio}

Este es el punto de partida para reflexionar sobre una segunda y consecuente afirmación. Una vez reconocida la dificultad antes expuesta sobre

1 Incluso en aquellas áreas donde el factor de unidad -o uno de ellos- se busca a nivel religioso, y se producen en consecuencia elaboraciones teológicas comunes, con esa intencionalidad política clara, que han de ser aceptadas en su organización política, se encuentran inflexiones y contradicciones ievitables por las mismas estructuras.

12 BONNET (1988: p. 9) habla de los politeismos como parte de una lógica clasificatoria de los seres divinos, en la que cada divinidad se define en relación con las otras, pero no estáticamente, sino a través de la posición que ocupa en cada momento.

13 Cuyo carácter acumulativo se explica por una mentalidad no excluyente, y son intrínsecas a la estructura de una realidad subcomunicada, tradicionalista por la continuidad que imprime el carácter cohesivo de los ritos que articulan cada comunidad o unidad de convivencia, etc.

14 Sin ser otra cosa que un marco más para delimitar el juego de factores y su área de acción, ni imponga una constricción absoluta. 
los panteones fenopúnicos, BONNET (id: p. 9), se remite a los planteamientos de P. Xella (1986). Para éste, los politeismos fenicios representan una «fase di avanzata disgregazione " ${ }^{15}$ respecto de los cananeos del II milenio ${ }^{16}$. El fraccionamiento político fenicio, en pequeñas ciudades que son un microcosmos en si (BONNET id. p. 4), explica las formas de la religión fenicia. Ésta se convierte asi en factor de diferenciación frente a otras comunidades.

Después de años buscando una interpretación comparada ${ }^{17}$ de las religiones sirias-palestinas tras los hallazgos de Ugarit-Ras Samra, se llegó a la ruptura, marcada por las advertencias de Caquot (1983) y Sznycer (1981/a). Situándose en el extremo opuesto, se afirma la no evidencia de relación entre religión cananea y fenicia, aparte de los teónimos que sobreviven, como no la hay de que los textos ugaríticos correspondan a una organización ritual equivalente (COOPER 1987/a: p. 35 ss).

Afirmación contundente para una documentación que bascula entre la procedente de Ugarit y la Biblia, y los escuetos textos epigráficos fenicios o arameos. $Y$ excesiva si contrastamos la información y su discontinuidad, su carácter aislado y parcial, como todos han reconocido. Por tanto, la primera cuestión es la ya tan manida de si conocemos los panteones del área siriopalestina del II y I milenio con la suficente profundidad y a lo largo de un número suficiente de lugares como para establecer una comparación adecuada y como para afirmar tal estado de coherencia mayor en los primeros.

Este creemos debe ser justamente el contexto de las susodichas advertencias de Sznycer (1981/a) y Caquot (1983). Una percepción que consideramos más acertada sería la de dos estadios sucesivos, en el que posiblemente haya que introducir un sesgo geográfico-espacial, y en el que la religión fenicia sea el último apéndice de un mundo cananeo que hemos de suponer lleno de variantes en sí. Sólo una laxa generalización como la explicada a través de una lengua y de una consiguiente "lógica común" se. puede aplicar a un conjunto tan heterogéneo.

Por tanto tales «deformaciones ciudadanas» bien pudieran estar ya presentes en el II milenio. Forman parte de esa «lógica cultural» marcada

15 Hace esta afirmación a propósito de la conveniencia de postular la existencia de tradiciones de tipo mitológico, y se remite a su vez a un trabajo anterior: XELLA, 1981, p. 11.

16 El mismo XELLA (1983: p. 401) se refiere a unas "trasformazioni che ne provocano la disgregazione e tendono a frammentare pantheon prima omogenei ed organicamente articolati, in una serie di culti poliadi incentrati sostanzialmente su singole divinità, dai caratteri "regali» fortemente enfatizzati e che vedono concentrarsi in esse la maggior parte delle prerogative e delle funzioni".

17 Que dió como consecuencia, en parte, un desarrollo conjugado como el de MESLIN DU BUISSON (1963). 
por el individualismo e independentismo de cada comunidad ${ }^{18}$. La descomposición del politeismo cananeo en uno «residual» fenicio que perdura en el I milenio, señalado por la falla del 1200, y apreciable en la proliferación de dioses sanadores, «parejas de divinidades políadas", etc, debe sopesarse.

El primer punto a considerar es la referida ruptura del 1200 a. C. Siendo señalada, debe matizarse, sobre todo en sus continuidades, frente a las indudables rupturas. Para el caso fenicio, lo es casi exclusivamente en la información ${ }^{19}$, y en sentido positivo: en la aparición de la documentación propiamente "fenicia». Pero ha sido cuestionada por Baurain (1986) para otros materiales ${ }^{20}$. Indudablemente, en el I milenio se produce una descomposición general en el área oriental ${ }^{21}$, marcada por el anegamiento arameo de las culturas preexistentes. $Y$ es muy posible que haya una gradación en esa descomposición a lo largo de las sucesivas integraciones políticas y culturales ${ }^{22}$. Pero las tradiciones fenicias (GRAs, ROUILLARD, TEIXIDOR 1989: p: 26) se refieren a traslados de población y nuevos reasentamientos tras la crisis del 1200 a. C.

Entendemos que la diversidad y el particularismo «urbanos" sostenida para los politeismos fenicios es igualmente aplicable a la cananea del II milenio ${ }^{23}$

18 Este es precisamente el sentido, entendemos, de las advertencias de estos autores -CAQUOT, SZNYCER-: no negar una cultura común, a valorar eso sí con sumo cuidado, para no caer en simples "trasposiciones".

19 RÖlLIG (1983: p. 378), subraya precisamente el carácter no arbitrario de esta fecha del 1200 a. C.

20 Así, este autor no aprecia diferencia suficiente en los marfiles de Kamin el-Loz entre antes y después del 1200 a. C. como para subrayar la ruptura. Reflexión que nos parece importante si entendemos la arqueologia de las ciudades fenicias en el 11 milenio. BONNET (id. p. 2) se hace eco de esta apreciación porque es esencial mantener la continuidad que pretende su argumentación para explicar el nombre de Melkart, vinculándolo con los dioses M/k que aparecen en el III milenio en Ebla.

21 López, Sanmartin (1993: p. 489 ss); Gras, Roulllard, Teixidor (1989: p. 27, 32-35, etc). Sobre los préstamos arameos en la lengua fenicia: Healey, F. J., Phoenician and the Spread of Aramaic, Att I Congresso Internazionale de Studi Fenici e Punici, vol. III, (Roma 1979) Roma, pp. 663-6, donde se revisan algunos de estos rasgos comunes, desde los de Biblos, Zinjirli, Karatepe, etc.

22 Babilónica, persa, helenística.

23 Por no hablar de diversidad de condiciones de vida más amplia todavía. Así, CaQuOt (1983: p. 10), escribe que "Los semitas occidentales, desde su aparición a principios del /l milenio a. C. vivieron en condiciones muy diversas: algunos de ellos conocieron la civilización refinada y compleja de las ciudades-estado, otras se mantuvieron por completo al margen de las zonas cultivadas del "creciente fértil". Resulta imposible pensar que esas diferencias no se vieran reflejadas en la plano religioso". $Y$ en tal, caso, ¿de dónde podría provenir esa mayor coherencia que se insinúa para el II milenio a. C. en sus panteones?, ¿de un mundo más "asentado", donde hubiese una mayor cantidad de contactos interiores y menores presencias exteriores? No es esa la impresión que produce la presencia desde inicios del II milenio, primero de infiltraciones amorreas, la presencia hurrita en la zona nororiental primero, y luego la mitannia, más extendida y que se desarrolla en una organización política que comprende peiodicamente las regiones al occidente del 
tanto o más. El espacio sirio ha sido en el Il milenio un área abierta, políticamente fragmentada ${ }^{24}$ y sometida a potencias exteriores, donde difícilmente se ha podido producir una elaboración teológica conjunta destinada a dar coherencia religiosa a la región como si de un sólo estado se tratase ${ }^{25}$. Tan sólo se vincula - repetimos- por las importantes percepciones que impone una lengua y una lógica cultural común. Luego el politeismo fenicio hemos de suponerlo inserto en una realidad análoga y sujeto a circunstancias no muy diferentes de las existentes en el II milenio ${ }^{26}$; y no como una forma evolucionada o degenerada, sino como una supervivencia adaptada, más allá de acontecimientos de carácter no radicalmente distintos de los del milenio anterior.

Observamos así que existen testimonios de una continuidad «importante» en ciertas divinidades, apreciables en Resef ${ }^{27}$, como botón de muestra, pero también en los casos de Mekal ${ }^{28}$, etc. ${ }^{29}$. Aun cuando la pervivencia misma de los teónimos es un elemento problemático, como plantea la misma $\mathrm{C}$. Bonnet ${ }^{30}$ en sus consideraciones metodológicas.

Finalmente, la no coherencia política entre las ciudades fenicias (CoOper 1987(b), p. 312; Xella 1986: p. 32), o la identificación de variantes dialectales entre cada ciudad ${ }^{31}$ no implica la no existencia de un panteón común más o menos coherente, de acuerdo a los principios enunciados en el primer punto, relativo a los politeismos y áreas culturales ${ }^{32}$.

Eufrates. Para continuar con la hitita, especialmente en Siria septentrional, y la egipcia, en el extremo merdional. Es en ese momento donde se aprecian importantes modificaciones en las religiones cananeas, con fenómenos como los hapiru y la formación del estado de Amurru.

24 Excepción hecha de la unificación bajo el reino de Qatna en los primeros siglos del II milenio.

25 Incluso en tal caso encontraríamos manifiestas las referidas contradicciones características de los politeismos antiguos.

${ }_{26}$ Ya que las circunstancias ambientales o estructurales no son muy diferentes de la señalada para el ll milenio, y su radicación es cananea. Así lo apuntan los indicios de supervivencias de divinidades que presentaremos a continuación, o el contraste con el las manifestaciones de las religiones arameas que encontramos en el resto del área sirio-mesopotámica desde el I milenio.

27 POPE \& ROLLIG 1965: p. 305-6

28 POPE \& RÖLLIG 1965: p. 298-9

29 CAQUOT (1983: p. 12), por ejemplo, hace referencia a una divinidad Hawran en el teóforo "Hawranabi" documentado en Mari; dios que reaparece en el siglo XIV en Ras Samra: donde asume las funciones de un dios médico y en el siglo VIII a. C., en un amuleto fenicio hallado en Arslan Tash: y en una inscripción griega de Delos, donde se le menciona como Auroras en compañia de Heracles, como dios de la ciudad Palestina de Jammia (actuál Yavné). Cf infra para el caso del Milkom ammonita.

30 Id. (p. 8-9) cuando hace una observación importante, que nosotros usaremos: se refiere al ejemplo de la presencia de Resef en Ebla, Ugarit, Emar y Fenicia: muestra el carácter de longevidad del teónimo: pero seguramente no de la misma divinidad a lo largo de milenios, con su misma fisonomía, etc, .... y habla, asi mismo, de hacer la historia de "Melkart, de los melkares" (p. 9).

31 G. Garbini, 1977, I dialetti del fenicio, Aion 37, pp. 283-94, y referencia en Rollig (1983: p. 378-9).

32 Un caso semejante seria el de los griegos, tan celosos siempre de su independencia, y sin embargo con una cultura más o menos común. Se trata, en suma, de la cuestión arriba planteada 
La segunda parte de la afirmación es la relativa a la tendencia identificada en los panteones urbanos a reducirse en favor de una "pareja» de divinidades. El enunciado de esta «tendencia» es cuidadoso. Xella (1986: p. 33) reconoce que puede deberse a los sesgos de la documentación, o a una "traditión «formulaire" stéréotypée». Y para Bonnet (id. p. 4) tienen un carácter esencialmente cultual. Pero parece que de acuerdo con esta idea, de forma más o menos explícita, se «necesita» justificar la presencia de divinidades como Esmún junto a Melkart tal y como aparece en el tratado de Asarhaddon ${ }^{33}$.

Acabamos de hacer referencia al fenómeno de la continuidad de las divinidades documentadas en los milenios anteriores a la Edad del Hierro y que vuelven a aparecer a lo largo de todo el mundo fenicio. Aun asumiendo el problema de los cambios que éstas hayan tenido a lo largo de tiempo y lugares distantes, la dispersión dentro del área de expansión fenicia-tiria de divinidades como las citadas de Resef, Mekal, pero también las de una geografía común fenicia, y no necesariamente "políada", como Bal-Hammon ${ }^{34}$ Tanit y Pumai ${ }^{35}$, etc., nos hablan reiteradamente de un panteón común.

En el caso concreto de Tiro tenemos varias referencias que apuntan en esta dirección. Estas empezarian en el II milenio a. C., con la posible mención en la epopeya de Kirta del santuario de "Atirat de Tiro, diosa de los

entre configuraciones políticas e institucionales, y de los papeles jugados en ellas precisamente por la religión como elemento de identificación. Por otra parte, en Fenicia se han reconocido diferentes etapas de hegemonía y autonomía política de unas ciudades sobre otras, con intercambios de población, etc (GRAs, Rouillard, TeIxidor 1989: p. 26).

33 BONNET (id. p. 41) trata de justificar esta presencia por las circunstancias políticas concretas: las del sometimiento de su ciudad poliada, Sidón, a los asirios, y la apropiación por Tiro de su divinidad protectora. Pero como veremos más abajo a propósito del mito, y como pasaremos a argumentar ahora, Esmún no aparecería necesariamente como dios políado de Sidón, sino como compañero de Melkart, al que se hace referencia en el mito citado por EuDOxO DE CNido (ap. Atenaios, IX, 45) y Zenobio (Centur, V, 56) como Heracles y lolaos (en otros casos, se identificaría con Asclepios). Cf. la misma BONNET en la p. 111, nota 389. Sobre el mismo: cf. FuLCo 1987.

${ }^{34}$ Para la continuidad de los Baales (hasta el caso de Baal-Beq, los de Hatra, etc, o de Aserat). Tenemos, así mismo, la continuidad del culto de Baal Hammon, el Señor de la montaña del Amanus, en los confines septentrionales de la costa siria, culto identificado claramente como cananeo, documentado en los primeros testimonios fenicios procedentes de Biblos (GRAS, ROUILLARD, TEIXIDOR 1989, p. 36).

35 En Sarepta, y en el mundo cartaginès (Cf. GRAS, ROUILLARD, TEIXIDOR 1989: pp. 43 ss). Se añade la referencia de P. Bordreul (1987) Tanit au Liban, St Phoen V, pp. 79-86. Otro aspecto que trataremos más abajo es la relación con Astarté como parte de esa problemática. Sobre las continuidades de divinidades en el área Siria, se pueden aportar datos por ejemplo Se inciuye igualmente a considerar como parte e un panteón común el caso de la estela de Nora (Cerdeña), dedicada a Pumay, con fecha del siglo IX, ya que no se identifica en ningún panteón ciudadano. 
sidonios ${ }^{36}$. Siguen con la referencia al Zeus ${ }^{37}$, al que dedica Hiram I una columna de oro, y con el conjunto de divinidades mencionadas en el tratado referido de Asarhaddon. $Y$ terminan con la mención de una estatua de "Apolo» que aparece en el relato de Plutarco ${ }^{38}$ a propósito del sitio de Tiro por Alejandro, con lo que estamos ante la pista de otra divinidad, distinta de Melkart. Son todos datos que nos hacen pensar en una comunidad de divinidades presentes en la ciudad junto a Astarté y Melkart, componiendo un panteón en toda regla.

Si se entiende esta "pareja políada» identificada en las ciudades fenicias y Tiro como una adscripción localista y cúltica (asociada al papel que asume el monarca, pero también un sacerdocio, dentro de la comunidad, y a una determinada organización ritual), y no como una esclerotización de la idea de un panteón común, no parece haber ningún problema en conjugar estos datos señalados con los dioses políados.

Estamos sin duda ante teocracias y a ellas se adscriben figuras protectoras de la monarquía ${ }^{39}$, o de las comunidades ciudadanas, de raigambre local. Pero ello no implica que sean desconocidas o ajenas a otros lugares, resultado tanto de la expansión mediterránea, en la que se han podido encontrar elementos fenicios de diferentes lugares que han participado -quizás como sometidos o asociados ${ }^{40}$ - en la expansión, como de tradiciones comunes.

Melkart aparece en suma asociado a una diosa principal, y éste es el rasgo principal y común denominador de todos estos cultos; igualmente, el teónimo Milkastart, semejante al empleado en otros lugares como Sidón y Biblos, nos habla una vez más de una «lógica común», que aparece igual en Biblos con Adonis (POPE \& RöLLIG 1965: p. 234), Sidón con Esmún, etc (POPE \& RÖLLIG 1965: p. 286-7), tema tratado también insistentemente ${ }^{41}$.

36 CoOper (1987: p. 44) la da por cierta. Del Olmo (1981: p. 255 y 275 nota 120, donde incluye un listado de las referencias), recoge la posibilidad de otras localizaciones

37 De la referencia de Flavio JosEFo (Contra Apión I 117-9), y la corrección de Menandro sobre el segundo templo mencionado por Heródoto (II, 44), que estaría dedicado a Zeus (ARRIANo, II 24, 5). Sería Zeus Olímpico, o Baal Shamin, según BONNET (1988: p. 48 y nota 83: que envia a la p. 36).

3B Vid. Alex. 24; BONNET 1988: p. 53.

39 RoulllaAd, Gras, Teixidon 1989: p. 35;

$40 \mathrm{Cf}$. el sometimiento de las comunidades de Botris (actual Batroun) al norte de Biblos, por Tiro (Gras, RouILlaRd, TeIxIDOR 1989: p. 48-49), y las anteriores referencias sobre hegemonias, convivencias, etc entre estas ciudades (id: p. 26, etc).

${ }^{41} Y$ asi se reencuentra a lo largo del área de expansión tiria, tanto en Kitión, como en la mayor parte del mundo palestino. Las excepciones, como en Biblos, se deben sin duda a tradiciones muy antiguas, Astart es conocida en los textos de Ugarit, donde presenta un papel poco activo: mientras que en la documentación del I milenio aparece como figura dominante: pero -como 
De lo dicho hasta ahora se colige que pueda hablarse de un panteón tirio en el que, pese al predominio cultual de una "pareja de divinidades" documentada, debemos inferir por otros indicios que se aplican elementos de una lógica politeística no diferente del resto de las que conocemos.

Si entendemos que el mundo fenicio forma parte de tal «lógica común", en la que los indicios relativos a una conjunto común de divinidades y ritos resultan significativamente semejantes, hay que suponer que en Tiro ha funcionado esa "lógica clasificatoria» de manera semejante.

\subsection{El caso de Melkart y su inserción en una "lógica religiosa" y en una "estructura religiosa común"}

Se plantea entonces como se inserta dentro de los aspectos míticos y cúlticos la tradición del culto a los ancestros, y en concreto, a los ancestros reales ${ }^{42}$, problema central en el trabajo de Bonnet. El culto a los ancestros, reconocido desde el Neolítico como muy importante (CAUVIN 1972: p. $104 \mathrm{ss}$ ), se cruza con el de la institución monárquica, y con el de una "lógica clasificatoria", como decimos. Se añade además un problema de denominaciones sobre las divinidades, inserto en una percepción institucional jerarquizada del mundo, a las que tanto en el área fenicia y cananea, como en otros lugares, se le aplican epítetos como señor ( $b^{\prime} l ;$; ' $d n$, al conocido de Adonis), príncipe ("príncipe santo" = sr qds) ${ }^{43}, y$, por lo que sabíamos hasta ahora, también rey (m/k), que pasaban a denominar a la divinidad por completo. Dejaremos esta cuestión de momento, para tratarla en conjunto con las relativas a la institución monárquica (cf. infra).

El primer problema es la clara identificación de los Rephaim o rapau, su actividad y su vinculación con la monarquía.

El politeismo ugarítico, que es el más documentado, distingue entre esos ancestros reales, o epónimos, como parte de una lógica cultural que, de manera análoga a la griega, usa de la intervención humana. Pero eso sí, de una determinada categoria "social" de seres humanos. La categoría ctónica de los rp'aum que acoge al soberano difunto (XELLA 1986: p. 38) parece estar suficientemente diferenciada del resto de las divinidades, si

hipótesis- es posible que haya simplemente una repartición geográfica de los cultos. En el norte de Siria se mantiene el de Attart?/ Atirat, mientras que en el sur dominaria Astart.

42 Los conocidos como r'pm, o Rephaim en textos ugaríticos y Biblicos: POPE \& RÖLLIG 1965.

43 A Resef, en Sidón, donde se le llama "príncipe santo»: Cf. A. CAQUOT ACF, 1978, p. 575, referencia procedente de XELLA (1983: p. 404, nota 25); cf. también XELLA 1986: p. 36 y nota 22. 
bien se mantienen el problema de los dioses calificados de $r p^{\prime} u$, como Baal.

P. Xella (1986: p. 38) reconoce el silencio de las fuentes directas sobre tal aspecto del culto a los ancestros. Y la "tradiciones difusas" referidas a personajes sobrehumanos de carácter todavia dudoso a las que hace referencia Xella, en las que se habla de pasión/muerte y resurrección, tienen enfrente a divinidades claramente identificadas, tanto en el caso del mito ugarítico de Baal, o los conocidos de Osiris ${ }^{44}$.

Por otra parte, en una inscripción púnica tardía ${ }^{45}$, los Rephaim se identifican con los Manes. Por lo tanto, tenemos en primer lugar que el nombre se mantiene como tal, y que en la inscripción púnica se establece una correspondencia entre ambas clases de entes divinos muy significativa. Como en tantos otros casos de entidades divinas, pervive a lo largo de más de milenio y medio, desde que se documenta en Ras Samra.

$Y$ por otro lado, si esta lógica propuesta por $C$. Bonnet en la identificación de Melkart como un rap'u se aplica, hay que contrastarla con otros casos del área palestina del I milenio a. C., que se han valorado de manera semajente a la de Melkart, pero que en el caso del Milkom (Molech) ammonita, presenta, además, una significativa coincidencia en el nombre. Ammonitas, moabitas y edomitas (DIAKONOV 1991: p. 300) serian tribus de sedentarización reciente en Trasjordania procedentes del desierto de Arabia, y hay que estar bien advertidos que cada una de estas religiones "étnicas" o "nacionalistas», tiene sus propias idiosincrasias cultuales. El dios Kemosh, la principal divinidad moabita que aparece en la inscripción de Mesha ${ }^{46}$, parece tener ancestros en el dios Kamish mencionado en las listas de divinidades de Ebla (ca. 2500 a. C.) ${ }^{47}$.

44 El caso de la saga mejor comocida y más popular, la de Gilgames, presenta, significativamente, el hecho final de la muerte. Cf. EDzARD, en Haussig 1965, pp. 69-73. con las referencias correspondientes.

45 CAQUOT (1983: p. 25) "Mantener y desarrollar la vida parece hacer sido la aspiración esencial de los mitos de Ras Samra. Si alguna función se reconocia a los muertos era la de contribuir a la renovación de la vida. Los fragmentos de un poema que sin duda constituía la continuación de la leyenda de Aqhat parece atribuir el papel de restauradores de la vida a los misteriosos Rephaim, término que volvemos a encontrar en el siglo I en una inscripción púnica acompañado de su traducción latina: Ios manes". Cf. GRAS, RouILLARD, TEIXIDOR 1989: p. 192.

46 Circa 830 a. C. por el rey Mesha de Moab, para celebrar sus victorias sobre los israelitas (CAQUOT 1983: p. 26-7)

47 El párrafo del libro de los Jueces (11:24) ha sido interpretado como equiparando Kemos con Milkom, el dios principal de los ammonitas; y en esta interpretación se ha sugerido que Milkom fuese un título de Kemosh ("el rey"), y que en realidad fuese Kemos la divinidad adorada por moabitas y ammonitas. Igualmente aparece una divinidad Astar-Kemos, que se ha interpretado bien como ... (MatTINGLY 1987 pp. 1-3). 
Pero el caso de Milkom - divinidad principal a la que denominan con el nombre de rey, como Melkart- nos trae dos cuestiones respecto a nuestro caso:

a) La cuestión de la nominación entre los semitas de su gran dios como rey, como apunta Caquot ${ }^{48}$, costumbre documentada en otros pueblos (cf. infra).

b) La otra posibilidad sería que, efectivamente, se aplicase «de manera sistemática» el nombre de "rey" a los ancestros reales ${ }^{49}$, y que efectivamente tal título (malik - malikum) conformase el de Melkart y Milkom, ejemplificando ambas divinidades mecanismos y procedimientos comunes.

Nos resta por anotar otra tendencia en la aproximación de divinidades reconocidas como tales a los Rephaim. Es el trabajo de Xella (1983), iniciado con Ribichini, que propone encontrar una categoría de divinidades "sanadoras", con un trasfondo común en el culto a los antepasados, a los rapau. Estos aparecen en la composición del nombre de Sadrapa como $s d-r p '$ ', según propone Pope \& Röllig (1965: p. 287) en relación con su carácter sanador, con el que identifica Röllig (id.) el término rpu tal como aparece en el rpu $b^{\prime \prime}$ de los textos ugaríticos.

\subsection{Los rituales, el sacerdocio, el santuario y los mitos}

Estos aspectos que pasamos a enumerar, relativamente independientes, se conjugan y entrelazan de tal manera que presentan en alguna medida cierta resistencia para encajar sin más en la hipótesis de C. Bonnet ${ }^{50}$. Provocan, en cualquier caso, una reflexión sobre cuestiones por terminar de aquilatar (como la composición del ritual dedicado a Melkart).

Respecto de los rituales, en principio, y siguiendo a Lipinski (1970), la egersis consistiría en un rito de muerte y resurrección, acompañada esta última de una hierogamia. La comparación que hace Bonnet entre el ritual tirio de la egersis y otros del Cercano oriente ${ }^{51}$, le conducen a lo que es

48 Asi, escribe (1983: p. 26-7): "Ello nos permite constatar una de las escasas constantes que es posible encontrar en toda esta maraña de nombres divinos imposible de organizar: los diferentes pueblos semitas reconocieron todos siempre a su gran dios la condición de rey, y consecuentemente la conducción de la colectividad humana".

49 Identificados por lo demás en el resto de las fuentes como Rephaim

50 El ejemplo del sacerdocio asociado o no al monarca y el papel de la hierogamia asociados a la dinastia, o las noticias de Heródoto sobre la antigüedad del santuario tirio.

51 (id. p. 110), al insertar las prácticas rituales tirias "dans un cadre rituel d'ensemble auquel se rattache aussi". 
una variada gama de rituales y festivales semejantes, entre los que se combinan rasgos, y presentando aspectos que pueden relacionarse de alguna manera con el culto a Melkart, pero entre los que convendría también distinguir: Rituales de Año Nuevo, como el que describe Luciano de Samosata, que era un gran festival del fuego, (De Dea Syria, 49: BonNET id. p. 109-11). El akitu babilónico, compuesto igualmente de distintos elementos (fuego, ritual de inversión y "chivo expiatorio", hierogamia); las adonisias, etc ${ }^{52}$. Estamos, en suma, ante un ritual que forma parte de unos "tipos" orientales compuestos de elementos comunes, en el que la cuestión del desarrollo concreto tal y como ha sido formulado por Lipinski, sin dudar en ningún momento de sus aportaciones, permanece abierta; por ejemplo, en sus protagonistas.

Efectivamente, que todavía por dilucidar la cuestión del sacerdocio, que trata de refilón Bonnet (id. p. 112-113) en una argumentación a propósito de la realeza sagrada poco extensa, que puede dar lugar a otras apreciaciones ${ }^{53}$. Reconoce la existencia de un sacerdocio, y de un sacerdote principal. El conjunto de sacerdotes asociados al santuario y culto de Melkart se confirmaría por el relato de Heródoto ( $v$. infra.). Respecto del sacerdote principal, en el relato de Justino (XVIII, 4, 5) se identifica claramente la alteridad entre la persona del rey Pygmalion y la de su tio Acherbas, sacerdote principal de Melkart y segundo personaje de importancia en la ciudad. Aspecto importante que tiene que ver tanto con el ritual que se plantea en la egersis (¿quien es el actante en tal ritual?) como el papel que tiene en éste la figura del monarca y la articulación social en linajes - que afectan a los principios dinástico de la monarquia, como lo plantea Tsirkin ${ }^{54}$ - y por tanto, quizás a los sacerdocios, en su estudio,

52 Cf. el desglose clásico y esencial de Frankfort (1976: p. 284-352 inclusive) de todos estos temas: desde el peligro del servicio y el rey sustituto (pp. $284 \mathrm{ss}$ ); las fiestas estatales y las alegrias del servicio (p. 287 ss); la construcción de templos (p. 289); la unión rey diosa (316 ss); el rey como hijo de dioses (p. 320); la veneración de estatuas reales (p. $323 \mathrm{ss);} \mathrm{el} \mathrm{festival} \mathrm{del} \mathrm{año}$ nuevo (p. $333 \mathrm{ss}$ ), etc.

${ }_{53}$ Hace referencia además (p. 113, nota 399), de las reservas de J. ELAYI (1986) entre el carácter dinástico y poliado de Melkart, disociando ambos, mientras que C. BONNET las considera solidarias. La observación de Elayi es cabal dado que tal carácter dinástico no deja de presentar efectivamente problemas.

54 1990. Este autor se apoya en la anécdota referida por CuRcIO RUFO (IV, I, 1-20) y DIODORO (XVII, 47) tras la caida de Sidon (Diodoro situaría equivoadamente el episodio en Tiro) cuando Alejandro destrona al rey Straton (i.e. Abdastart) y comisiona a Hefestio para que elija otro rey; éste ofrece el trono a sus amigos notables por su riqueza (se supone que de origen aristocrático) pero estos rehusan puesto que de acuerdo con las costumbres, el trono sólo podia heredarse dentro de la familia real; y asi la puirpura recayo en un tal Abdalonim, quien pertenecia a la familia real sidonia, pero era pobre. 
enlazando con una reflexión entre la idea de "realeza sagrada" (Cf. infra BONNET: p. 112-113) y el papel real de la monarquía como encarnación de poderes frente al de la ciudad como cuerpo social que presenta un límite a la institución; comunidad coordinada por el rey, pero no sometida a él ${ }^{55}$. Evidentemente, si suponemos al rey de Tiro sumo sacerdote de Astarté, como en otras ciudades donde el rey parece ser sacerdote de la divinidad femenina principal ${ }^{56}$, y realiza él la hierogamia, ¿cuál es la función del sumo sacerdocio de Melkart?; ¿quien realizaba la hierogamia que formaría parte del ritual de la egersis?.

Cuando Heródoto (II 44) visita Tiro y habla con esos sacerdotes, éstos remontan la fundación del santuario a lo que arqueológicamente se ha demostrado es aproximadamente el inicio de la secuencia arqueológica documentada para Tiro (2.700 a. C.), y asociada a la ocupación del lugar ${ }^{57}$. En éste se produce una hierofanía por la asociación del árbol en llamas, según nos cuenta el relato de Filón de Biblos transmitido por Eusebio de Cesarea (P. E. I, 10, 10-11), y donde se erigirían por Ousoos las estelas o betilos que lo caracterizan en el relato de Heródoto y muestran las monedas (WILL 1950-51: MESNIL DU BUISSON 1963).

No insistimos en la adscripción del lugar, cuya identidad para el caso tirio o gaditano, como lugar de manifestación del dios es claro: esencialmente, la identificación del árbol con Astarté. etc (Así lo recoge WILL 195051). Tampoco en las referencias consiguientes que da Heródoto sobre el santuario de Heracles en Tasos, que sitúa cinco generaciones antes del Heracles griego, hijo de Anfitrión, y que nos remitiría a ese 11 milenio a. C. ${ }^{58}$.

Esto le da a Melkart un claro sentido topográfico, como lo tiene el aludido mito de la "ocupación de la ciudad", realizada por Oussos e Hypsuranios, pero en un lugar dedicado a la divinidad, donde se encuentran lo que serían sus símbolos asociados.

55 TSIRKIN (1990: p. 34) pone el ejemplo del mencionado tratado de rey Baal de Tiro con Asarhaddon, en el que se menciona a los hombres de la comunidad junto al rey.

56 Por ejemplo, en la inscripción de Esmunazor de Sidón (GRAS, ROUILLARD y TEIXIDOR 1989: p. 40) donde la reina es sacerdotisa igualmente de Astarté. GRAS, RouillaRd y TEIXIDOR (1989: p. 35) evitan en concluir que el rey sea siempre el gran-sacerdote de la divinidad local.

57 Otras noticias y cronologias las da Josefo (Antiquit lud. VIII, 3, 1), para quien Tiro se habría fundado 240 años antes de la construcción del templo, lo que da una fecha del 1200. Justino (XVIII, 3,5 ) sitúa la fundación un año antes de la guerra de Troya, cuya fecha tradicional es el 1193-84 según Eratóstenes. Gras, RouILLARD, TEIXIDOR 1989: p. 52, nota 15, Remiten a BLEGEN (The Cambridge Ancient History, II/2, 1975, p. 163).

${ }_{58}$ Momento en que ya se habla de Tiro en la documentación de Tell el-Amarna (Gras, ROUILLARD, TEIXIDOR 1989: p. 46). Sobre otras hipótesis y otras cronologías: Cf. GRAS, ROUILLARD, TEIXIDOR 1989: p. 49-50. 
La combinación de este carácter arcano y tópico, más el culto asociado a una divinidad femenina, semejante a los otros casos documentados en fenicia, como ya hemos expuesto, y con lo que sería un sacerdocio como institución cúltica constituida, debe servirnos para valorar el carácter de la "reforma» expuesta por Josefo (Antiqu. VIII 5, 3). Esta, como explicaremos más abajo en relación con al carácter de la monarquía tiria, se entiende posiblemente más como una "reinstauración» en una circunstancia política dada: lo que será, con Hiram, el principio de la hegemonía tiria.

Una última cuestión que parece quedar en el aire, entre un excepticismo ante la existencia de mitos (XELLA 1986: pp. 30-31) y el reconocimiento del carácter heroico de Melkart y también sus elementos míticos (BONNET 1988: p. 97), tema paradójicamente no desarrollado, es la posible identificación de elementos en el mito, que iría asociada a la argumentada inserción de Melkart junto a otras divinidades en el panteón.

Empecemos por la interpretación de la escena representada en el relieve conocido como la "natividad tiria», como la llama C. Bonnet (1988: p. 82 ss). La pieza fué publicada e interpretada por E. Will (1950-51) con anterioridad a las tratadas por C. Bonnet de H. Seyrig ${ }^{59}$, y la de B. ServaisSoyez ${ }^{60}$, contextuada en un Tiro helenizado, que le satisface más, no son las únicas. De hecho la argumentación sobre la identificación de la figura de Telefos arcadio, hijo de Heracles y Augías, siendo ingeniosa, parece menos sólida que la propuesta por E. Will. Este autor dedica una parte sustanciosa de su trabajo (pp. 3 a 9) a identificar el lugar en que se desarrolla la escena como el santuario tirio descrito en los relatos de Aquiles Tacio (Leucipo y Clitofón, II, 14: Anth. Pal. XIV, 34), Nonnos (Dionisíacas, 40, 422 ss), Heródoto (II, 44) y Filón de Biblos $(2,5-7)$, ya identificados por Mesnil du Buisson (1963) y Grotanelli (1972) en su estudio sobre los origenes duales de Tiro, y la misma Bonnet (1988). La asociación del árbol en llamas, con la serpiente y el águila no parece ser la accidental de diferentes elementos que pretende la interpretación de B. Soyez y sigue C. Bonnet. De acuerdo con esa ubicación de la escena, lo que se presenta es una clara "exposición» con amamantamiento. Este se realiza por una gacela o

59 H. SeYAig, Les grands dieux de Tyr à l'époque grecque et romaine, Syria 40, pp. 19-28, reconociendo la tríada tiria compuesta por Astarté, Zeus (simbolizado en el águila) y Melkart, al que una hipotética tradición fenicia haría que el niño fuese amamantado por un cérvido, interpretación que desecha $C$. BONNET por la carencia de fundamento de la "ancienne théorie des triades phéniciennes", razonada por XELLA (1981: p. 14).

60 Recherches sur le panthéon des principales cités pheniciennes aux périodes hellénistique et romaine (tesis doctoral inédita), Lieja, 1974, p. 269-275, referencia que da C. BONNET 1988: p. 83 , nota 253. 
cierva ${ }^{61}$. ¿Es ese uno de los caracteres identificables en la figura de Melkart, a la que se atribuye el señorío de un santuario identificable por los elementos como el descrito?

Otro fragmento esencial es el Eudoxo de Cnido (ap. Atenaios, IX, 45) y Zenobio (Centur, $V, 56$ ) en el que se describe la muerte de el rey heroico por Tifón, mientras era acompañado por lolaos/Esmoun. De nuevo, Bonnet ${ }^{62}$ duda de la originaria autenticidad de los personajes en el relato de la resurrección por el humo de las codornices asadas con Astarté.

Otro aspecto que encaja en el carácter de "héroe cultual» que debe entenderse tiene Melkart es su relación con el descubrimiento de la púrpura (BONNET id.: p. $74 \mathrm{ss}$ )

Son todos ellos rastros puntuales e incoherentes entre sí, entresacados de un conjunto de fuentes dispersas, recogidas todas por Bonnet, pero que deben contrastarse en conjunto si se afirma, como de hecho se hace, que Melkart ha tenido efectivamente una «leyenda heroica», como hipóstasis del soberano, pero se duda a la vez (XELLA 1986: ) de la vitalidad de tales narraciones en la cultura fenicia.

\section{LAS INSTITUCIONES Y LA HISTORIA: TIRO Y SU MONARQUIAA}

La hipótesis de la reforma de Hiram que convierte a un Rapaum, a un ancestro real, en dios de la ciudad tiene un carácter político incuestionable que ha de contrastarse con la institución monárquica tiria y con la circunstancia concreta en que nace. La primera cuestión es si efectivamente en el mundo cananeo y tirio la práctica de la divinización está lo suficientemente consolidada, y el problema que plantea una reforma religiosa de semejantes características.

Bonnet se remite sobre todo a el ejemplo de la divinización de los ancestros en Akkad, y al caso Egipcio, los casos más claros de divinización del monarca en el Cercano Oriente.

61 ThOMPSON, a propósito de Nergal ha identificado a la vaca o a la gazela como la diosa que lo amamanta (la Hera que amamanta a Heracles, la loba que amamanta a Rómulo).

62 BONNET (1988: p. 111, nota 389), anota que "se puede interrogar por la identidad de lolaos que hace quemar las codornices para Melkart. Algunos le han querido ver, en tanto que dios sanador, en correspondencia a Esmún. Sin embargo, es generalmente identificado con Asclepios. Aqui es más razonable pensar que lolaos ha sido incorporado al mito en tanto que fiel compañero de Heracles, y no como interpretación de una dios fenicio". 


\subsection{Las monarquias orientales y sus variantes}

La institución monárquica puede y debe considerarse de manera global para todo el Cercano oriente. Primero, para caracterizar sus rasgos comunes, y luego para contrastar los modelos propuestos por las áreas nucleares frente a la periferia. Esta visión ha de completarse a nivel de áreas culturales y momentos históricos, en las que diferentes circunstancias han impuesto sesgos a esa institución común (en particular la realidad política y su tamaño, las tradiciones locales, y las necesidades de emulación/ competencia entre estados).

En ese sentido es importante distinguir entre una monarquia -con un especialista intermediario encarnado en el monarca- y una forma teocrática; o, si este matiz en los términos es muy vago para el caso oriental, entre el carácter de la figura del rey divinizado presente en la tierra, como aparece en Egipto, por una cuidada elaboración teológica (cf. FrANKFORT 1976) y el vicario del dios (figura históricamente anterior), el intermediario entre la comunidad y el orden divino: un especialista que, por su capacidad para establecer el orden justo dentro de la comunidad, y su conocimiento de los ritos necesarios, sirve de intermediario entre el orden de los agentes causales últimos: aquellos que determinan el destino humano, los verdaderos dueños, "los dioses que eligen» (FrankForT, id: p. 260).

Parece clara la evolución de uno a otro en el caso mesopotámico, y se ha expuesto para el caso Egipcio hace tiempo (Frankfort 1976, p 39 ss). Se ha discutido sobre la evolución en el mundo mesopotámico del poder del "vicario del dios", del rey, hacia la divinización, y las rupturas consiguientes que se han producido ${ }^{63}$. Y está igualmente clara la relación entre esta evolución y el desarrollo de los imperios. Se trata en suma de una fórmula política desarrollada por los imperios como solución unificadora a la diversidad real que existía en ellos.

Pero el mundo ugarítico nos ha brindado en sus textos un cuadro para el área cananea a valorar con cuidado. Del Olmo toca el tema de la ideología regia en los fragmentos de la «Epopeya de Kirta» y en la "Epopeya

\footnotetext{
63 Por ejemplo, parece que de "vicario" se pasa a "hijo del Dios" -como en el Estela de los Buitres del Protodinástico III-; se diviniza claramente en la cultura acadia, con una serie de implicaciones que están por valorar (Frankfort 1976: p. 246 y ss); es posible que, efectivamente, haya un antecedente de "divinización" de los cabezas de familia que vayan a un "limbo" especial. $E$ inversamente, se ha planteado la descomposición o pérdida de esa caracter con la amorreización en el II milenio, durante los llamados reinos o estados regionales, para reaparecer con el mundo asirio.
} 
de Aqhatu". De la existencia de algunos rasgos de divinización ${ }^{64}$ plantea su carácter real o retórico. (1981: p. 279-81). La cuestión es -escribe Del Olmo- "si tal ideología se aplicaba "dogmáticamente" en Ugarit a todo rey, al estilo egipcio, o más bien es una hipérbole retórica, favorecida por la concepción y exaltación del rey como héroe epónimo, sin que ello implique la transmisión de tal prerrogativa de inmortalidad a sus sucesores; adviértase -continúa- cómo en Ugarit tal ideología regia de filiación divina e inmortalidad parece haber entrado en crisis y se encuentra expresada únicamente en fórmulas interrogativas, en las que resuena el contraste entre el "dogma» y la experiencia real».

"Dentro de la misma línea vemos como en Ugarit, por lo demás, la función regia no se agota en una dispensación mecánica de fecundidad al pais, sino que implica una actuación ético social como dispensador de justicia; en ese sentido su "enfermedad" no sólo pone en peligro el orden cósmico, sino también el social, en cuanto le incapacita para cumplir su función indispensable de "lider", "juez" $y$ "salvador" del débil. Resuena un eco claro de la ideología de Israel".

En la «Epopeya de Aqhatu» (Del OLmo 1981: p. 359-60) presenta la «ideología regia", encarnada en Daniilu "preocupado como Kirta por su descendencia, cumpliendo las mismas funciones de "dispensador de justicia" (...) que lleva a cabo "a la puerta de la ciudad, en la era". Para Del Olmo, en suma "el carácter "divino" del rey no aparece tan claramente enunciado como en la Epopeya de Kirta, ni se habla de su inmortalidad. Más bién, ésta queda excluida por Aqhatu como posible suerte de los "mortales". Resaltan más, en cambio, las características morales: el exacto cumplimiento de sus deberes sociales, religiosos y paternales".

Del Olmo (1981: p. 363-4) identifica finalmente el sentido del poema de Aqhatu con el del cantar de gesta, y a los Rapauma con héroes, encarnaciones del pasado hecho leyenda épica, que concreta la imagen religiosa y social ideal del grupo en que se conservan. Son héroes regios, eso si, en contraste a los patriarcas bíblicos, que son héroes tribales. Sin implicar necesariamente una proyección cúltica ${ }^{65}$, serian parte de una realidad ideológica semejante a la proporcionada por otros relatos.

Tampoco en el mundo fenicio encontramos restos claros de una divinización del monarca, ni aparece el carácter «heroico" de manera señalada

64 Como hijo, pero también "mancebo" o criado, y siervo del dios $/ / \mathrm{u}$

65 Como proponen algunos autores que recoge DEL OLMO, en la nota 116: ejemplo, DrESSLER, AT, 638-42, 660-71: el triple posible Sitz im Leben: cúltico, dinástico, pedagógico. 
en ningún momento, ni hay nada en el contexto cultural que destaque tal aspecto, ni que supongamos ha de hacerlo. Las inscripciones de los sarcófagos, desde el de Ittobaal, no hacen alusión a tal divinización. No deja de ser factible, empero, la hipótesis de que Hiram, "organizador del imperio comercial de Tiro" ${ }^{66}$ acometiese tal reforma, y la mantuviesen algunos de sus sucesores, quizás hasta Jeremías. Pero muchos problemas surgen a la hora de identificar al rey con un dios vivo.

El último aspecto a considerar es central en la propuesta de Bonnet: el problema de denominar epitéticamente a una divinidad como "mlk», rey, está detrás de toda la argumentación, y decidir la viabilidad de aceptar el paso "m/k»: el significado tradicional de rey o la argumentación como «a los señores", en relación con los problemas de la realeza tiria. Esta última cuestión a propósito de tal hipótesis está en el nombre mismo de la divinidad: «malik» - "qart": "antepasados reales" "de la ciudad", según la propuesta de Bonnet. Se trata de aplicar el sustantivo "rey" (que por otra parte es equivalente a Baal) ${ }^{67}$ por un "teóforo" compuesto por esta palabra que se identifican con la ciudad. Los antecedentes en que se apoya es * la documentación de un teóforo acadio en la que aparece el nombre y * un teóforo malik en un culto eblaita a los "malik» (que pueden identificarse con «los señores»).

La ideología monárquica se mezcla con la "mítica" o teológica, y el orden cósmico y social se entienden así como gemelos. De ahí que se compartan instituciones, y, naturalmente, nombres. La argumentación es compleja y ambigua a la vez, y empieza con la identificación misma del sentido del ejercicio del poder divino y el terrestre. Desde Sumer encontramos el problema de unas titulaturas compartidas entre hombres y dioses. La identidad del sustantivo "en" (señor) en las titulaturas de los gobernantes (sumos sacerdotes, dentro del problema que han presentado entre en, lugal, etc) y como parte del teonimo En-ki, o En-lil. El "señorío" del rey de la tierra - sólo como vicario de la divinidad-y el "señorio" "divino" se comparte por un problema lingüístico, que se ha traspuesto de distintas maneras a diferentes lenguas en relación con los epítetos dados a los dioses, titulaturas creadas para resaltar su posición que resultan del proceso histórico de jerarquización de las sociedades.

Pero es realmente al argumentar que, tipológicamente hablando, el nombre de Mlkrt corresponde - como heredero de una tradición- al dios-

66 Si se nos permite la expresión, admitiendo la caduca discusión sobre una talasocracia fenicia. (señor) como se documenta en los bilingües de Malta 
rey de una ciudad ${ }^{68}$ cuando se nos presenta lo que consideramos realmente la clave de la composición del nombre y del sentido de la divinidad.

Se trata, en ese sentido, tanto de la integración del politeismo local en un conjunto coherente de divinidades dentro de un cultura, como del fenómeno de justificación de un poder "teocrático", en el que la monarquía puede estar puesta bajo la protección de una divinidad

La siguiente argumentación que da (BONNET id. p. 419), sobre la presencia en la antroponimia acadia del elemento teóforo de Malik y en Egipto, donde el faraón es la encarnación son dos casos reconocidos de divinización del soberano que es posible no tengan demasiados equivalentes en las teologias teocráticas de oriente, y que se explican por procesos históricos muy concretos. El acadio tiene que ver con el primer intento de organización imperial en Mesopotamia; el egipcio, con el esfuerzo dado por reunir los nomos en un solo estado, esfuerzo que se prolonga en la historia de Egipto a lo largo de éxitos y crisis, para lo que se ayudaron de distintas teologias, una de las cuales fue al parecer la de Osiris-Horus.

\subsection{El carácter de la actuación religiosa de Hiram}

En suma, la explicitación de la lógica histórica particular introducida en la general del área, tanto el panorama religioso de las ciudades fenicias, con una percepción corregida en la relación "pareja políada"-resto del panteón fenicio, como otros puntos particulares (antigüedad del lugar sacro, carácter del culto, semejanza con otras divinidades etc) permiten una reconsideración global de la cuestión propuesta para Melkart.

Una revisión de la circunstancia concreta en que se establece la egersis y de la posterior historia de Tiro y de su hegemonia nos lleva a plantear la acción de Hiram como una posible acción política de carácter independentista. Menandro de Efeso y Justino $(18: 3,5)$, señalan que la ciudad de Tiro

68 Reproducimos el párrafo de BONNET (1988: p. 418) "Ceci explique sans doute la régulière qualification des dieux poliades comme lugal + toponyme (9). Typologiquement parlant, Melqart se presente comme l'héritier de cette tradition du dieu-roi d'une ville. II en est meme l'expression par excellence tant par son nom que par son titre de Baal de Tyr. II est intéressant, dans cette perspective, de relever l'existence en Mésopotamie d'un correspondant sémantique parfait de Melqart, dlugal-uru, qui apparaît dans quelques documents de Girsu et dans des listes d'offrandes à Fara et que l'on a propose d'identifier à Ningirsu, le dieu poliade de la ville de Girsu (10). Dans ce cas, le titre de "Roi de la Ville" ne désigne pas une entité divine autonome, comme lest Melqart, mais sert d'epiclese. Malgré le parallélisme entre ces deux "Rois de la Ville", il n'y a aucune raison de croire que Ningirsu, s'il s'agit bien de lui, ait pu constituer un antécédent historique de Melqart. Nous avons affaire, plus simplement, à une logique culturelle similair. 
fue "fundada» por sidonios el año 1191 a.C; se insinua así la posibilidad de que, tras su destrucción, Tiro fuera reconstruida -o rehabitada - bajo los auspicios de Sidón, ciudad que, según toda la evidencia, fue el centro mas importante del sur de Fenicia durante los siglos XII y XI a.C ${ }^{69}$. En ese momento Tiro no aparecen en absoluto en la documentación que sí menciona a Biblos y Sidón, hasta el reinado de Hiram I (969-936 a.C.) (AUBET 1987: p. 28). Con él se inicia la gran expansión tiria, que exigiria cambios institucionales importantes, empezando por zafarse, muy posiblemente, de la autoridad sidonia. Existió sin embargo un estado Tiro-Sidón (AUBET 1987: p. p. 29).

El acto de Hiram, como parte de una independencia respecto de Sidón, puede significar tanto una simple reanudación de un culto olvidado - reentendiendo en la expresión de que se realiza por "primera vez" tal egersis, es decir un acto piadoso, posiblemente semejante a otros de restauración (lo hace el príncipe sidonio Esmounazor II (Gras, Rouillard, Teixidor 1989: p. 40) pero está dentro de las tradiciones míticas, desde las de Gilgames, las acadias, las de Gudea, ... : ir al bosque a cortar cedros, restaurar los templos, ... restaurar el culto). Pero puede implicar también una reforma religiosa de tal envergadura, como la argüida por Bonnet, que enmascare o soporte una reforma política relacionada con su naciente hegemonía sobre los tráficos de los alrededores.

El carácter continuista podría estar apoyado tanto en las referencias cronológicas dadas por Heródoto sobre el santuario tirio, como sobre las comentadas a propósito del santuario de Heracles tasio. La existencia misma de un santuario y su culto supone una continuidad, que se sobreentiende si se acepta la veracidad del relato herodoteo y de los propios sacerdotes tirios, a los que se les supone un acceso a unos archivos remotos. En todo caso, si admitimos la tranformación de una figura preexistente -asociada con el santuario ya fundado-implica un acuerdo de todo un sacerdocio.

De acuerdo con esta reforma, se plantea el problema de la asociación de Melkart a la expansión, no necesariamente adquiriendo un carácter marino, como se ha "transmitido" en base a una hipótesis de Dussaud (194648), sino quizas, como plantean Gras, Rouillard y Teixidor (1989) en relación a la organización de los intercambios. Ya que son los barcos del rey, y ningún otro, los encargados de acometer semejante empresa.

69 (AUBEt 1987: p. 26-7). A su vez, señala Aubet, esta situación explica el hecho de que, en época tardia, Sidón reivindicara todavia en sus monedas la ciudad de Tiro como su hija. "Sidón, madre de Cartago, Hippo, Citium y Tiro", rezan leyendas monetarias de época helenistica. 
El silencio mencionado relativo a la vinculación entre los rapau, el Baal con carácter de rapau, y Melkart, por una lado, hacen del argumento de Bonnet más sugerente y sutil que contundente en lo que a este punto se refiere.

Si efectivamente tuviésemos una reordenación general de divinidades «menores», como Esmún y Melkart, esto no parece óbice para que se incluyan en un panteón en el que aparecen divinidades anteriores de manera clara.

\section{CONCLUSIONES}

El trabajo de C. Bonnet, eminentemente positivo y muy sugerente, plantea un nuevo campo de realidades enlazando con problemas emergidos en la investigación de la religión cananea y feno-púnica, retomando cuestiones tratadas por una investigación clásica como la de $\mathrm{H}$. Frankfort, trasvasada a otros ámbitos espaciales. El mundo del culto funerario y de los ancestros, olvidado y redescubierto, así como la relación con la monarquía, si corre el peligro de ser un fondo de saco, inidentificado respecto al resto de las manifestaciones cultuales, forman un trasfondo alusivo importante.

Pero la argumentación desarrollada, tratando de hilar problemas muy diferentes, desde el nombre, a la historia, nos enfrenta con ciertas dificultades. Este trabajo, que nace a la sombra de las aportaciones de la investigación de $\mathrm{C}$. Bonnet, aspira a enfocar la cuestión tal y como ha quedado tras ese avance, mostrando sus irregularidades. La ubicación de Melkart como hipóstasis del soberano, entendida como una aportación, debe ahora explicarse en relación con las instituciones y la religión tiria y fenicia. Si es una aportación importante, no deja de ser una hipótesis "bien argumentada». $Y$ adolece de una confirmación más precisa.

Ese mismo asunto del nombre, la conversión de un «ancestro" de carácter heroico en dios, las cuestiones que se plantean inmediatamente alrededor de la institución dinástica que es capaz de propiciar tal reforma y que debiera contrastar con una monarquía de carácter urbano y controlada por otras intituciones de manera importante -aspectos no excesivamente desarrollados, creemos-, así como las referidas al control y organización de la expansión que ha podido pasar por varias fases, en la que ha podido tener que ver la institución monárquica de una manera semejante a otros casos, o ha sido desarrollada por instituciones sociales son temas por rematar. 


\section{BIBLIOGRAFÍA}

Albright, W. F., 1975: Syria, the Philistines and Phoenicia, The Cambridge Ancient History, II: 2, pp. 507-536.

Aubet Semmler, M. Eugenia, 1987: Tiro y las colonias fenicias de Occidente, ed. Bellaterra, Barcelona.

Baurain, Cl., 1986: Portées chronologique et géographique du terme "phénicien", St. Phoen. IV, p. 7-28, Namur.

BonNET, Corinne, 1988: Melqart. Cultes et mythes de l'Héraclès tyrien en Méditerranée, Studia Phoenicia VIII, Lovaina-Namur.

Tzavellas-Bonnet, Corinne, 1983, "Le dieu Melqart en Phénicie et dans le bassin mediterranéen: culte nationel et officiel" Studia Phoenicia / y /I. 2, p. 195 ss O.L.A. Leuven.

BordreulL, P., 1986: “Attestations inédites de Melqart, Baal Hamon et Baal Saphon à Tyr (Nouveaux documents religieux phéniciennes II)", Studia Phoenicia IV. Religio Phoenicia. Namur. Acta Colloquii Namurcensis, (14-15 dic. 1984). pp. 77-86.

BrElich, Angelo, 1977, "Prolegomenos a una historia de las Religiones", en Historia de las Religiones siglo XXI, vol. 1. Las religiones Antiguas I. Madrid, siglo XXI, pp. 30-97.

BRELICH, Angelo, 1958: /l politeismo, Roma.

CAQuOT, André, 1983: Las religiones de los semitas occidentales, en Las religiones antiguas. vol II, Historia de las religiones, dir. por Henri-Charles Puech, ed. siglo XXI, Madrid.

Cauvin, Jacques, 1972: Religions Neolithiques de Syro-Palestine. Documents, Paris.

Cooper, Alan, M., 1987(a): Canaanite Religion: An Overiew, en The Encyclopedia of Religion, vol 3, dir. por Mircea Eliade, pp. 35-45, Chicago.

COOPER, Allan M., 1987(b): "Phoenician Religion", en The Encyclopedia of Religion, vol 10, pp. 311-18.

DaHOOD, M.-J., 1958, “Ancient Semitic Deities in Syria and Palestine», en MOSCATI, S. (ed.), Le antiche divinità semitiche. (Studi Semitici 1,) Roma. pp. 65-94.

Del OLmo LETE, 1981: Mitos y leyendas de Canaan según la tradición de Ugarit, Madrid.

DE MEYER, L., 1986: "ll vinico oriente dalla bataglia di Qadesh alle invasioni dei Popoli del Mare": Le origine dei greci. Dori e mondo Egeo. (Musti, ed), Bari.

DetTIENE, M., 1986: Du polytheisme en général, CP 81, pp. 47-55.

DuSSAud, René, 1957: "Melqart, d'après récents travaux", RHR, $C L /(151)$, pp. 1-21.

Dussaud, R., 1946-48: "Melqart", Syria 25, pp. 205-30.

DusSAUD, R., 1947: "Astarté, Pontos et Baal", CRAI, pp. 201-24.

EHRENBERG, Victor, 1969: The Greek State, Methuen, Londres.

ELAY, J., 1986: "Le roi et la religion dans les cités phéniciennes à l'époque perse", Studia Phoenicia IV. Religio Phoenicia. Namur. Acta Colloquii Namurcensis, (14-15 dic. 1984). pp. 249-62.

FRANKFORT, H., 1976: Reyes y dioses, Madrid.

FulCo, W. J., 1987: "Melkart" en The Encyclopedia of Religion, vol 9, p. 365.

FulCo, W. J., 1976: The Canaanite God Resef, New Haven.

Gras, M., Rouillard, P., TeIxIDOR, J., 1989: L'Univers Phenicien, Artaud-CNRS, Paris.

Grottanelli, C., 1972(b): “Eracle dattilo dell'Ida. Aspetti "orientali», OA 11, pp. 201-8.

GrotTANELLI, Cristiano, 1972(b): "ll mito delle origine di Tiro: due "versioni" duali", OA 11, pp. 49-63.

Grottanelli, Cristiano, 1973: “Melqart e Sid fra Egitto, Libia e Sardegna", RSF 1, p. 15364 SS.

GrottANELLI, Cristiano, 1981: "Santuari e divinitá delle colonie d'Occidente", en La Religione fenicia. Matrici orientali e sviluppi occidentale. Atti del colloquio in Roma, 6 marzo 1979, Roma, pp. 109-33.

LIPINSKI, E., 1970, "La fète de l'ensevelissement et de la résurrection de Melqart", en Actes de la XVII RAI: Rencontre assiriologique Internationale. pp. 30-58.

López, Jesús, Sanmartín, Joaquín, (1993): Mitología y Religión del Oriente Antiguo. I. Egipto Mesopotamia. ed. Ausa, Sabadell.

Mattingly, Gerald L.: Moabite Religión, en The Encyclopedia of Religion, vol 10, dir. por Mircea Eliade, pp. 1-3, Chicago 1987.

MESNIL DU BUISSON, Robert du, 1963: "Origine et évolution du panthéon de Tyr", Revue de IHistoire des Religions, CLXIII, pp. 133-63. 
POPE, Marvin H., y Rölling, Wolfgang, 1965: “Syrien. Die Mythologie der Ugariter und Phönizier", en: HaussıG, H. W. (ed.), Wörterbuch der Mythologie. Götter und Mythen im Vorderen Orient Band I. Stuttgart, p. 217-312.

RIBICHI, S. - XELLA, P., (1979): Milk'astart, $m \mid k(m)$ e la tradizione siropalestinese sui Refaim: ASF, 7, p. 155 ,

RÖlling, W., 1982: Die Phonizier des Mutterlandes zur Zeit der Kolonisierung, en NIEMEVER, $H$. G. (ed), Phonizier im Westen, pp. 15-30, Maguncia.

Rölling, W., 1983: The Phoenician Language: Remarks on the Present State of Research, Atti I Congresso Internazionale de Studi Fenici e Punici, vol. II, (Roma 1979) Roma, p. 375-85.

SZNYCER, Maurice, 1981(a): "Les religions des semites occidentaux", en Dictionnaire des mythologies, Paris, pp. 421-95.

SZNYCER, Maurice, 1981(b): «Pheniciens et puniques", en Dictionnaire des mythologies, Paris. pp. 250-6.

TSIRKIN, YU. B., 1991: Phoenician and Greek Colonization, cap. 17 de Early Antiquity, M. Diakkonov, y Philip L. Kohl (eds), Chicago.

TSIRKIN, YU. B., 1990: Socio-political structure of Phoenicia. Gerión, 8 - pp. 29/43.

WiLL, Ernest, 1950-51: Au sanctuaire d'Héraclès a Tyr: l'olivier enflammé, les stèles et les roches ambrosiennes, Berytus 10, pp. 1-12.

Xella, Paolo, 1981: Aspetti e problemi dellindagine storico-religiosa, en La Religione Feninia. Matrici orientali e sviluppi occidentali. (Atti del Colloquio in Roma 6 marzo 1979), Roma, pp. 7-25.

Xella, Paolc, 1983: "Sulla piu antica storia di alcune divinitá fenicie" , Atti I Congresso Internazionale de Studi Fenici e Punici, vol. II, (Roma 1979) Roma, p. 401-9.

Xella, Paolo, 1986: "Le polythéisme phénicien", Studia Phoenicia IV. Religio Phoenicia. Namur. (Acta Colloquii Namurcensis, 14-15 dic). 1984. pp. 29-39.

ZWI WerblowSky, R. J., 1987(a): "Anthropomorphism”, en Enc. Rel, vol 1, pp. 316-20.

ZWI WeRBlowsky, R. J., 1987(b): “Polytheism”, en Enc. Rel, vol 11, pp. 435-9. 